\title{
KCNJ11 downregulation stimulates cardiac cell apoptosis in myocarditis
}

\author{
Seungmin Choi ${ }^{1,2}$, Joo Hee Chung ${ }^{2}$, Myung-Hee Nam ${ }^{2}$, Eunjung Bang ${ }^{3}$, \\ Jong Bok Seo ${ }^{2, *}$ and Sung-Gil Chi ${ }^{1, *}$ \\ ${ }^{1}$ Department of Life Sciences, Korea University, Seoul 02841, Korea \\ ${ }^{2}$ Korea Basic Science Institute, Seoul Center, Seoul 02841, Korea \\ ${ }^{3}$ Korea Basic Science Institute, Western Seoul Center, Seoul 03759, Korea
}

One of the inflammatory heart diseases, viz. acute myocarditis, occurs due to cardiac cell death. However, the molecular mechanism underlying cell death remains largely unexplored. In this study, we report that the down-regulation of KCNJ11, a central subunit of the ATP-sensitive potassium (KATP) channel plays a key role in the reduction of blood glucose, and is involved in apoptosis of cardiac cells. Using proteomics analysis of experimental autoimmune myocarditis (EAM), we show that the KCNJ11 level marked by decreased, whereas Camk2a expression increased significantly in EAM tissues by 16 and 20 days, compared to control tissues. Using ${ }^{1}$ H-MAS NMR we also show that glucose levels were slightly elevated in EAM tissues. In vitro assays using $\mathrm{H} 9 \mathrm{c} 2$ cardiac cells revealed that both lipopolysaccharide (LPS) and high glucose treatment decreased cell viability, in which toxicity was attenuated by treatment with KATP pharmacological openers, but not by the KATP blockers (Gli and 5-HD). Apoptosis induced by LPS or high glucose treatment was suppressed by $\mathrm{Ca}^{2+}$ chelator (BAPTA-AM) treatment. We found that KCNJ11 levels had decreased in cardiac cells by LPS or high glucose treatment, and siRNA-mediated knockdown of KCNJ11 expression further stimulated the LPS- or high glucose-induced apoptosis. Together, our results demonstrate first that KCNJ11 is down-regulated under inflammation and high glucose conditions and its inactivation facilitates cardiac cell apoptosis. We assume that down-regulation of KCNJ11 has an effect on the development of myocarditis.

Keywords: Apoptosis, blood glucose lipopolysaccharide, cardiac cells, myocarditis.

INFLAMMATORY heart disease, including myocarditis, is caused by infection of virus and protozoa, autoimmune responses and drugs ${ }^{1-3}$. Acute myocarditis is a heart disease that cannot be predicted because several clinical courses are asymptomatic or a variety of symptoms ${ }^{1,2}$. Acute myocarditis is induced by massive cardiac cell death, in which the accumulation of fibres results in fibrosis and

\footnotetext{
*For correspondence. (e-mail: sjb@kbsi.re.kr; chi6302@korea.ac.kr)
}

heart $\operatorname{attack}^{1,4}$. However, the signalling pathway of cardiac cell death remains largely elusive.

One of the well known myocarditis models is experimental autoimmune myocarditis (EAM), in which myosin injection triggers autoimmunity in heart tissues. The rat EAM shows elevated levels of toll-like receptor 4 (TLR4), caspase-3 cleaved form and tumour necrosis factor- $\alpha$ (TNF- $\alpha$ ), proof that rat EAM mimics viral myocarditis ${ }^{1,5,6}$. The TNF- $\alpha$ related apoptosis-inducing ligand (TRAIL), Fas (CD95/APO1) and TNF- $\alpha$ cell death receptors can control cell apoptosis and also trigger intrinsic apoptosis pathway ${ }^{7-9}$. Previous studies showed that mitochondrial $\mathrm{Ca}^{2+}$ stimulates an increase in reactive oxygen species (ROS) and induces mitochondrial permeability and apoptosis ${ }^{10,11}$. High levels of glucose are also known to trigger mitochondrial dysfunction, caspase activation and apoptosis ${ }^{12,13}$.

The ATP-sensitive potassium channels (KATP) regulate insulin secretion in pancreatic beta cells ${ }^{14,15}$. The octameric complex containing the Kir6.2 (KCNJ11) tetramer at the centre is the KATP channel, surrounded by four SUR1 subunits, which responds to glucose concentration via its opening/closing for regulating blood glucose levels ${ }^{16-18}$. Under high glucose conditions, the KATP channel is closed, resulting in cell membrane depolarization, $\mathrm{Ca}^{2+}$ uptake and apoptosis ${ }^{16,19,20}$. Studies also showed that inactivation of KCNJ11, the central subunit of the KATP channel, is implicated in cardiac cell death and heart diseases ${ }^{21,22}$.

To explore the mechanistic basis of cardiac cell death leading to acute myocarditis, we analysed the proteomics profiles using rat EAM model. We show that the KCNJ11 level is downregulated during acute myocarditis and its inactivation facilitates cardiac cell apoptosis triggered by inflammation or high glucose-induced stresses.

\section{Material and methods \\ Experimental autoimmune myocarditis induced by myosin in rats}

The EAM was induced by injecting $1 \mathrm{mg} / 100 \mu \mathrm{l}$ of porcine heart myosin and the Freund's adjuvant to footpad of 
seven-week-old Lewis rats $(n=15)$ twice on the zeroth and sixth day, in addition to injecting $500 \mathrm{ng}$ of Bordetella pertussis toxin intraperitoneally on the first day. The cardiac tissues were obtained at 9, 16 and 20 days after the first injection. The vehicle without $B$. pertussis toxin or pig heart myosin was injected into the control group $(n=5)$. All animal procedures were performed with permission from the 'Institutional Animal Care and Use Committee at the Korea Basic Science Institute' (KBSIAEC1602) ${ }^{23}$.

\section{Reagents treatment and $\mathrm{H} 9 \mathrm{c} 2$ cardiac cell culture}

Under condition $5 \% \mathrm{CO}_{2}$ humidifier at $37^{\circ} \mathrm{C}$ and incubator, the cells were cultured in culture flasks. The cells were obtained from rat embryonic heart tissue (ATCC, Manassas, VA, USA). Penicillin-streptomycin (P/S) 1\% and foetal bovine serum (FBS) $10 \%$ were added to Dulbecco's modified Eagle's medium (DMEM, Gibco, Basel, Switzerland $)^{23}$. Cytotoxicity was activated under glucose or LPS conditions. The reagents (Sigma-Aldrich, St. Louis, USA) were used in the treatment of cardiac cells at $37^{\circ} \mathrm{C}$ for $0,6,18$ and $24 \mathrm{~h}$. Diazoxide (DZ), glyburide (Gli), pinacidil (Pin) and 5-hydroxy decanoic acid (5-HD) were purchased from Santa Cruz Biotechnology (Santa Cruz, CA, USA) ${ }^{23}$.

\section{${ }^{1} H$ HR-MAS NMR analysis in EAM heart tissue}

${ }^{1} \mathrm{H}$ HR-MAS NMR analysis in EAM heart tissue was done according to previous experiments ${ }^{23}$. A $4 \mathrm{~mm}$ diameter zirconia rotor with inserts (total volume of $40 \mu \mathrm{l})$ was used, where $5-10 \mathrm{mg}$ of heart tissue samples was placed $^{23}$. Distilled water containing $0.05 \%$ (wt) of 3-(trimethylsilyl)-propionic- $d_{4}$ acid (TSP) to provide ${ }^{2} \mathrm{H}$ field locking was used to fill the rest of the rotor volume ${ }^{23}$.

The ${ }^{1} \mathrm{H}$ HR-MAS NMR spectra were recorded (Unity Inova 500 spectrometer; Agilent Technologies Inc., USA) under the operating conditions at 500.13 for ${ }^{1} \mathrm{H}$, a spin rate of $2300 \mathrm{~Hz}$, and temperature of $283 \mathrm{~K}$ and $4 \mathrm{~mm}$ gHX nano-probe head was attached for acquisition ${ }^{23}$. The Carr-Purcell-Meilboom-Gill (CPMG) spin-echo pulse sequence was used to collect the ${ }^{1} \mathrm{H}$ NMR spectra with total spin-spin relaxation time of $300 \mathrm{~ms}$ and spin-echo delay $(\tau)$ of $400 \mu$ s (refs 23,24 ). For each sample, 128 transients into $32 \mathrm{~K}$ data points were collected through a spectrum width of $8004.8 \mathrm{~Hz}$, an acquisition time of $2 \mathrm{sec}$ with water pre-saturation during the relaxation delay. A line-broadening function of $0.3 \mathrm{~Hz}$ was used to multiply the spectra and was then phase-adjusted, Fouriertransformed (FT) and manually baseline-corrected, and referenced to TSP $(0.00 \mathrm{ppm})^{23}$. Signal assignments for representative tissue samples were achieved using heteronuclear single quantum correlation (HSQC), total corre- lation spectroscopy (TOCSY) and two-dimensional ${ }^{1} \mathrm{H}-{ }^{1} \mathrm{H}$ correlation spectroscopy (COSY), the $500 \mathrm{MHz}$ library from Chenomx NMR suite version 6.0 and compared with the literature ${ }^{23,25,26}$.

\section{Protein level analysis in heart tissues and cardiac cells}

The total proteins of cardiac cells and EAM heart tissues were extracted in the RIPA buffer. Next, 10-15\% SDSPAGE gel was used to separate the $20-30 \mu \mathrm{g}$ of total proteins and then transferred to a polyvinylidene difluoride (PVDF) membrane. The primary antibodies for GAPDH (CST \#2118), cytochrome $c$ (CST\#4272), caspase-3 (CST \#14220), $\beta$-actin antibody (CST \#3700) and Kir6.2 (KCNJ11; PA5-26218) were purchased from Thermo Scientific (Waltham, MA, USA), Cell signalling (CST, Danvers, MA, USA) and Santa Cruz Biotechnology (Santa Cruz, Calif. USA).

\section{$m R N A$ analysis in heart tissues and cardiac cells}

Total RNA was extracted from EAM hearts tissues or the $\mathrm{H} 9 \mathrm{c} 2$ cardiac cells using $0.2 \mathrm{ml}$ of TRIzol reagent (Thermo Scientific) and converted to cDNA using the First Strand cDNA Synthesis Kit (Thermo Scientific). The qRT-PCR reaction was duplicated in $10 \mu \mathrm{l}$ of cDNA diluted 10 times in water using SYBR-Green PCR Master Mix (Thermo Scientific). Using Step One Plus Real-Time PCR System (Thermo Scientific), the qRT-PCR working conditions were as follows: $3 \mathrm{~min}$ at $95^{\circ} \mathrm{C}$ followed by 40 cycles at $95^{\circ} \mathrm{C}$ for $15 \mathrm{sec}$ (denaturation), $60^{\circ} \mathrm{C}$ for $25 \mathrm{sec}$ (annealing temperature), and $72^{\circ} \mathrm{C}$ for $25 \mathrm{sec}$ (extension). The 18S rRNA or GAPDH level was used to normalize the quantification of PCR products of the target genes. The primers used were-Camk2a sense primers: 5'CGAGGGCCTGGACTTTCA-3', and Camk2a antisense primers: 5'-TGCACGGGCTTGCTGTT-3'; GAPDH sense primers: 5'-GGCAAGTTCAACGGCACAGT-3' and GAPDH antisense primers: 5'-TGGTGAAGACGCCAGTAGACTC-3'; 18s rRNA sense primers: 5'-CATTCGAACGTCTGCCCTAT- $3^{\prime}$ and $18 \mathrm{~s}$ rRNA antisense primers: 5'-GTTTCTCAGGCTCCCTCTCC-3'.

\section{siRNA transfection into $\mathrm{H} 9 \mathrm{c} 2$ cells}

The cardiac cells were seeded in six-well plates without antibiotics. A transient transfection with lipofectamine 2000 (Invitrogen, Waltham, MA, USA) was applied when the cells were $60-75 \%$ confluent and the experiment was in accordance with the manufacturer's instructions ${ }^{23}$. In this experiment, siRNA (10 pmol) and lipofectamine $2000(2.5 \mu \mathrm{l})$ were individually diluted in $125 \mu \mathrm{l}$ serumfree Opti-MEM (GIBCO/Invitrogen) and incubated at 
$25^{\circ} \mathrm{C}$ for $5 \mathrm{~min}$, and then combined. The siRNALipofectamine mixture was incubated at $25^{\circ} \mathrm{C}$ for $20 \mathrm{~min}$ and added to the cell culture in a $5 \% \mathrm{CO}_{2}$ humidifier at $37^{\circ} \mathrm{C}$ for $16 \mathrm{~h}$. The next day, PBS was used for cell wash and the cells were cultured in fresh medium with $1 \%$ antibiotics. At 24, 48 and $72 \mathrm{~h}$ after transfection, the cells were harvested. The siRNA knockdown constructs used in the study were obtained from Cosmogenetech (Seoul, Korea) and their sequences are listed here: Kcnj11-rat335 sense primers: $5^{\prime}$-CCGCUUCGUGUCCAAGAAATT$3^{\prime}$ and antisense primer: 5'-UUUCUUGGACACGAAGCGGTT-3'; Kcnj11-rat-689 sense primer: 5'-GCAGAAUAUCGUAGGGCUATT- $3^{\prime}$ and antisense primer: $5^{\prime}$ UAGCCCUACGAUAUUCUGCTT-3'; Kcnj11-rat-1384 sense primer: 5'-GCAUCUCUCCGGAUUCCUUTT-3' and antisense primer: 5'-AAGGAAUCCGGAGAGAUGCTT-3'. The random sequence control mixtures were also provided by the manufacturer and treated as instructed.

\section{Cell viability assay in $\mathrm{H} 9 \mathrm{c} 2$ cells}

The MTT proliferation assay kit (ATCC) was used for cell viability analysis, according to the manufacturer's instructions $^{23}$. The $10 \%$ FBS/DMEM medium was used to culture cardiac cells in 96-well plates for $16 \mathrm{~h}$. At the end of the treatment, $10 \mu \mathrm{l}$ of MTT reagent $(0.5 \mathrm{mg} / \mathrm{ml} \mathrm{MTT})$ was treated to cells with or without the exposure of siRNA constructs, LPS or glucose, which was then incubated in a $5 \% \mathrm{CO}_{2}$ humidifier at $37^{\circ} \mathrm{C}$ for additional $4 \mathrm{~h}$.

\section{Statistics}

At least three independent experiments were performed to obtain the mean \pm standard deviation (SD) of the data. The statistical significance of the differences was confirmed using an unpaired Student's $t$-test. The SPSS Statistical Software version 24 (IBM, Armonk, NY, USA) was used for statistical analysis and one-way analysis of variance (ANOVA) was used to determine the $P$ value ${ }^{23}$. A $P$-value of less than 0.05 was considered statistically significant $(* *)$.

\section{Results}

\section{Apoptosis induction in EAM heart tissues}

To characterize cardiac cell apoptosis involved in myocarditis pathogenesis, we used the rat EAM model in which myocarditis was induced by myosin injection. Following injection of myosin or adjuvant (control) into rats, the EAM heart tissues were analysed on days 9, 16 and 20. As expected, myosin-induced EAM lesions (discoloured surface) were clearly seen at day 20 postinjection (Figure $1 a$ ). To assess apoptosis induction, we performed immunoblot analyses of cleavage caspase- 3 and cytochrome $c$, which are elevated through activation of the mitochondrial pathway of apoptosis. Compared to control tissues, EAM tissues at day 20 showed markedly increased levels of both cytochrome $c$ and cleaved caspase-3, confirming that active apoptosis is accompanied by myocarditis development (Figure $1 b$ and $c$ ).

\section{Elevated levels of glucose and KCNJ11 down-regulation in EAM tissues}

Previous studies demonstrated that the inactivation of KATP channel, a key regulator of calcium and glucose, is associated with the development of heart disease ${ }^{21}$. In the present study, ${ }^{1} \mathrm{H}$ HR-MAS NMR revealed that glucose levels were increased in EAM tissues, compared to control (Figure $2 a$ ). We thus measured the level of KCNJ11, a subunit of the KATP channel which controls calcium transport in response to glucose levels. An immunoblot assay showed that the level of KCNJ11 protein gradually decreased in EAM (Figure $2 b$ and $c$ ). In addition, we observed that the mRNA level of Camk $2 \mathrm{a}\left(\mathrm{Ca}^{2+}\right.$ associated calmodulin) gene was significantly increased in EAM tissues compared to control (Figure $2 d$ ). Given that elevated calcium in mitochondria activates mitochondrial apoptosis signalling, the results suggest that myocarditis pathogenesis might be associated with the elevation of glucose and calcium, and subsequent mitochondrial dysfunction and apoptosis.

\section{KATP openers attenuate cardiac cell death induced by LPS or high glucose}

To confirm whether abnormal KATP function is associated with cardiac cell death, we tested the effects of KATP opener (diazoxide (DZ) and pinacidil (Pin)), KATP-blocker glyburide (Gli) and 5-hydroxy decanoic acid (5-HD) or $\mathrm{Ca}^{2+}$ chelate (BAPTA-AM) on the cell viability of $\mathrm{H} 9 \mathrm{c} 2$ cells exposed to LPS or high glucose levels. MTT assay showed that compared to cells treated with LPS or glucose alone, cells co-treated with DZ $(100 \mu \mathrm{M})$ or Pin $(50 \mu \mathrm{M})$ exhibited $19-26 \%$ increase in viability (Figure $3 a$ and $b$ ). Conversely, cells co-treated with Gli $(100 \mu \mathrm{M})$ or 5 -HD $(100 \mu \mathrm{M})$ exhibited $43 \%$ reduction in viability compared to LPS- or glucose-treated control cells. Cells treated with BAPTA-AM showed 17$24 \%$ increase in viability compared to control (Figure $3 a$ and $b$ ). Figure $3 c$ and $d$ shows the effects of KATP openers, blockers or $\mathrm{Ca}^{2+}$ chelate BAPTA-AM on cell death. Consistent with MTT assay results, cells treated with DZ, Pin or BAPTA-AM were protected against LPS or glucose alone compared to control cells. 
Rat hearts (days after injection)

(a) 9

16

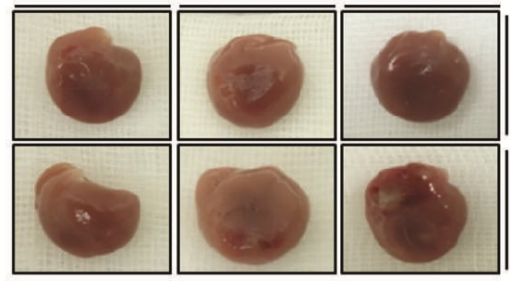

(b)

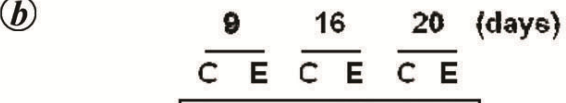

Control

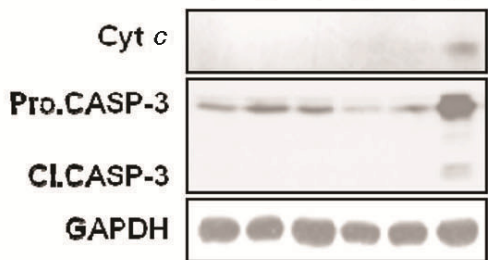

(c)
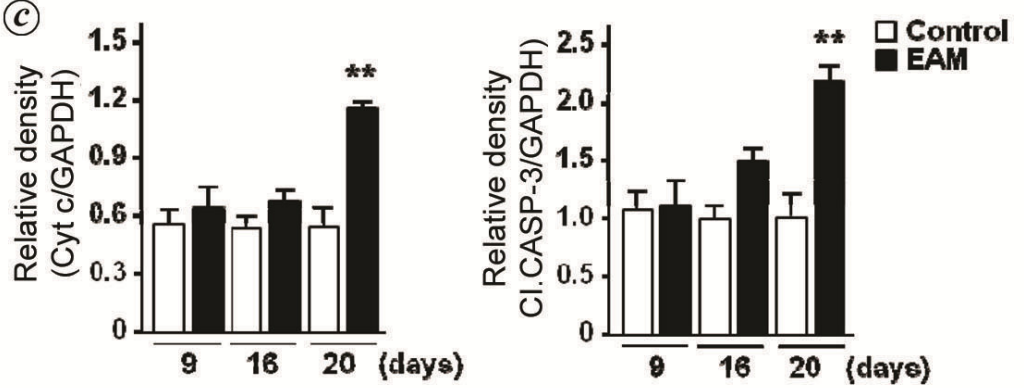

Figure 1. Changes in heart morphology and in apoptosis gene in the experimental autoimmune myocarditis (EAM) model during EAM induction. $\boldsymbol{a}$, EAM rat heart on days 9, 16 and 20. $\boldsymbol{b}$, Protein levels of cytochrome-c and cleaved caspase-3 proteins EAM heart. $\boldsymbol{c}$, Quantitative confirmation of cytochrome- $c$ and cleaved caspase-3 protein levels confirmed in EAM heart at 20 days. Data represent mean $\pm \mathrm{SD}(n=3) .{ }^{*} P<0.05,{ }^{*} P<0.01$ in two-tailed Student's $t$-test.
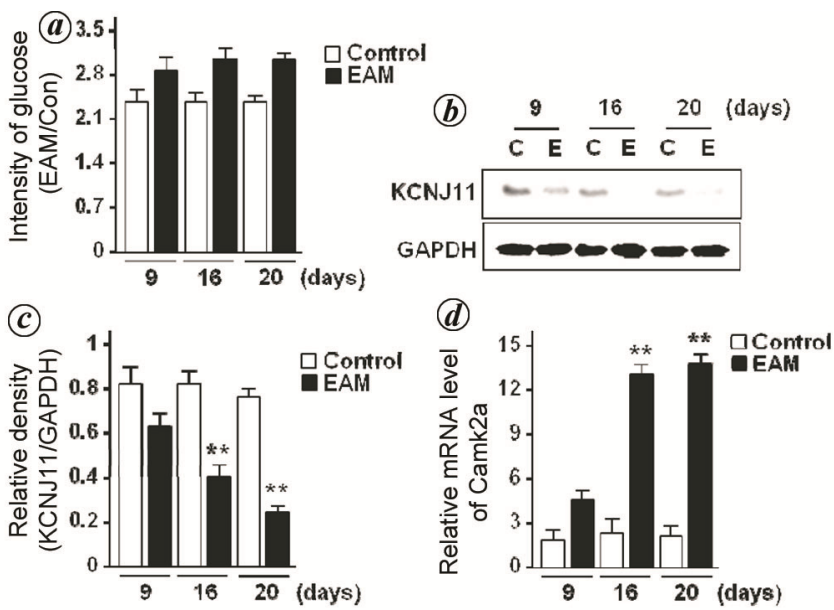

Figure 2. Changes in glucose levels by ${ }^{1} \mathrm{H}$ HR-MAS NMR and KCNJ11 and Camk2a levels in EAM model. $\boldsymbol{a}$, Changes in glucose level by ${ }^{1} \mathrm{H}$ HR-MAS NMR in EAM heart tissue. $\boldsymbol{b}$, Western blot showing KCNJ11 protein levels at 9, 16 and 20 days in the EAM model. $c$, Quantification of KCNJ11 protein levels confirmed on days 9, 16 and 20 in the EAM heart. $\boldsymbol{d}$, EAM cardiac tissue confirming the level of Camk2a mRNAat9, 16 and 20 days. Data represent mean \pm SD $(n=3)$. $* P<0.05,{ }^{*} P<0.01$ in two-tailed Student's $t$-test.

\section{KCNJ11 reduction facilitates cardiac cell apoptosis induced by LPS or high glucose levels}

We tested whether reduced expression of KCNJ11 was involved in cardiac cell apoptosis. Western blot assay showed that LPS or glucose treatment downregulated KCNJ11 expression in cardiac cells, which was accom- panied by an increase in cleaved caspase-3 levels (Figure $4 a$ ). A drastic decrease in KCNJ11 was observed in cells treated with LPS, compared to those treated with glucose (Figure $4 b$ ). We also observed that the mRNA level of Camk2a was increased in cells treated with glucose or LPS for $24 \mathrm{~h}$ (Figure $4 \mathrm{c}$ ). Moreover, siRNA-mediated knockdown of KCNJ11 expression further promoted glucose- or LPS-mediated apoptosis (Figure $4 d$ and $e$ ).

\section{Discussion}

Pro-inflammatory cytokines such as TNF- $\alpha$ play an important role in cardiac cell apoptosis triggered by pathogen invasion and inflammation ${ }^{5,27,28}$. It has been also reported that LPS-exposed heart injury is related to mitochondrial dysfunction and cell death in the EAM model $^{29,30}$. However, the molecular mechanism underlying cardiac cell death during myocarditis development remains unknown. In this study using myosin-induced rat EAM model, we have identified that KCNJ11 downregulation is implicated in induction of heart cell apoptosis under inflammatory conditions.

It is known that lactate levels are elevated in EAM tissues and increased lactate levels are associated with heart attack $^{31,32}$. Lactate moves to the liver and produces glucose through gluconeogenesis, and this glucose is again used as an energy source for muscles (Cori cycle). It is known that high levels of glucose cause mitochondrial dysfunction $^{33}$. In response to high glucose levels, the 
(a)

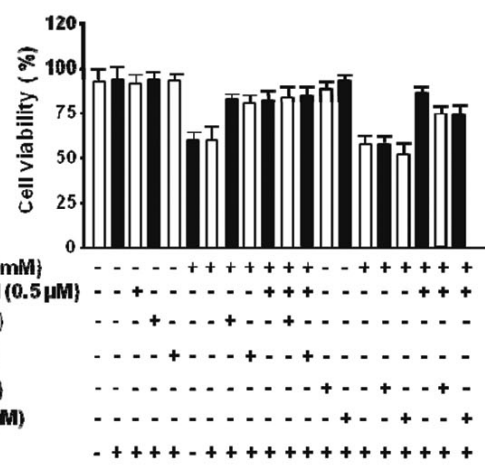

(b)
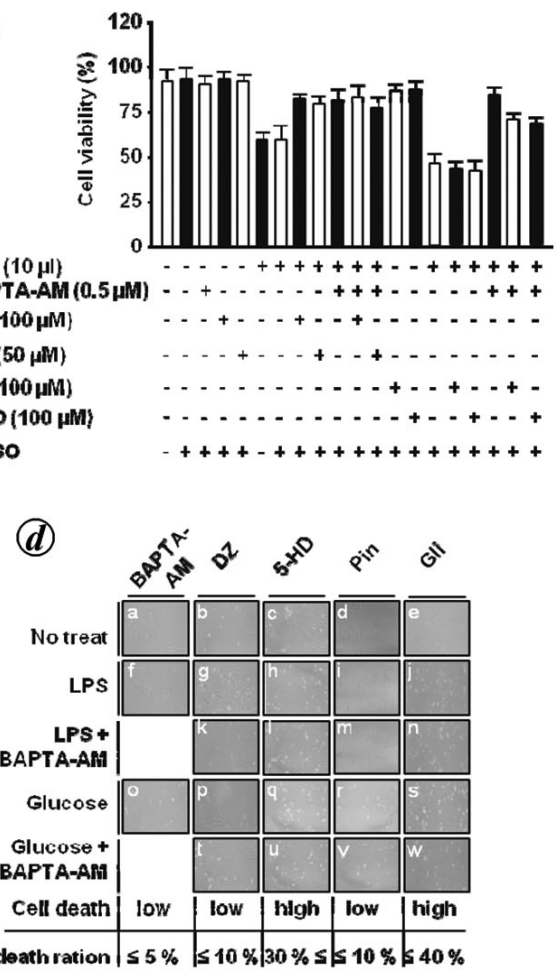

Figure 3. Cell viability assay by treatment with diazoxide (DZ), glyburide (Gli), pinacidil (Pin), 5-hydroxy decanoic acid (5-HD) and $\mathrm{Ca}^{2+}$ chelator (BAPTA-AM) on H9c2 cells under lipopolysaccharide (LPS) or high glucose conditions. $\boldsymbol{a}, \boldsymbol{b}$, MTT analysis confirmed that the cells treated with glucose or LPS showed change in cell viability. However, glucose or LPS toxicities were significantly by $50 \mu \mathrm{M}$ Pin or $100 \mu \mathrm{M}$ DZ or $0.5 \mu \mathrm{M}$ BAPTAAM. Glucose or LPS toxicity was found to increase with $100 \mu \mathrm{M}$ Gli or $100 \mu \mathrm{M} 55$-HD. $\boldsymbol{c}, \boldsymbol{d}$, Cells were treated without or with glucose or LPS, and $100 \mu \mathrm{M} \mathrm{DZ}, 50 \mu \mathrm{M}$ Pin, $100 \mu \mathrm{M}$ Gli, $100 \mu \mathrm{M}$ 5HD or $0.5 \mu \mathrm{M}$ BAPTA-AM to confirm cell morphology. Data represent mean $\pm \mathrm{SD}(n=3)$.

(a)

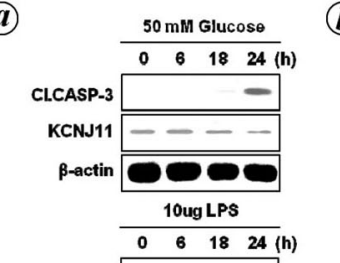

(c)

\begin{tabular}{rl|} 
CI.CASP-3 & \\
KCNJ11 & \\
B-actin &
\end{tabular}
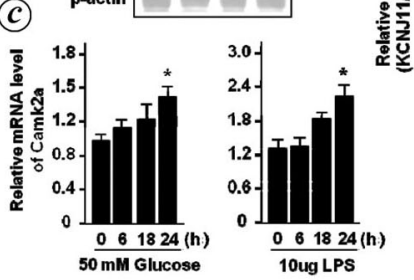

(b)
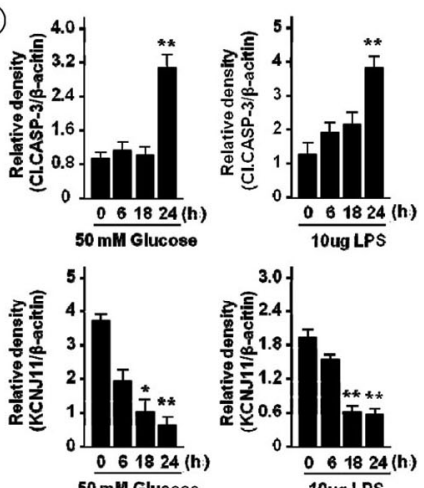

(e)

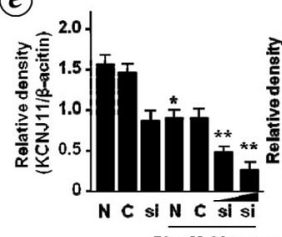

$50 \overline{\mathrm{mM} \text { Glucose }}$
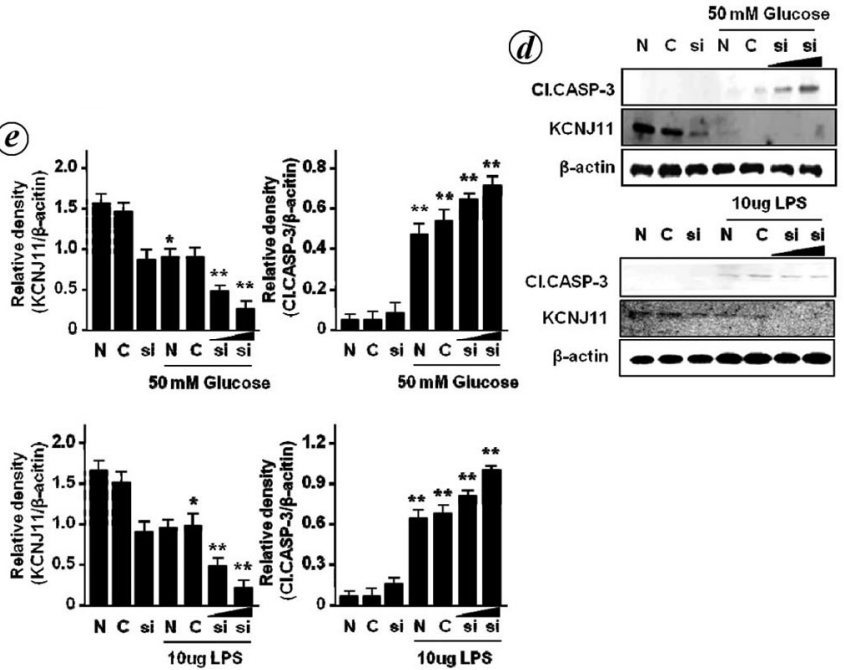

Figure 4. The exposure of LPS or glucose induces cleaved caspase-3 gene expression and the knock-down of KCNJ11 induces cytotoxicity of LPS or glucose in H9c2 cells. $\boldsymbol{a}$, In Western blot, the levels of KCNJ11 and cleaved caspase-3 protein were identified in H9c2 cells after 10 $\mu$ g LPS or $50 \mathrm{mM}$ glucose exposure. $\boldsymbol{b}$, In protein quantification, cleaved caspase-3 levels increased in $50 \mathrm{mM}$ glucose or $10 \mu \mathrm{g}$ LPS for $24 \mathrm{~h}$, while KCNJ11 levels decreased in 24 h. $\boldsymbol{c}$, Checking the Camk2a mRNA level in cells using qRT-PCR while being exposed to glucose or LPS for 24 h. $\boldsymbol{d}$, In immunoblot, $50 \mathrm{mM}$ glucose or $10 \mu \mathrm{g}$ LPS treatment in the cells induced the expression of cleaved caspase-3 and siRNA of KCNJ11increased the glucose or LPS effects. The loading control was $\beta$-actin. $\boldsymbol{e}$, Quantification of caspase-3 and KCNJ11 protein levels in H9c2 cells with siRNA. In the quantification of cleaved caspase- 3 and KCNJ11 proteins in $\mathrm{H} 9 \mathrm{c} 2$ cells, $50 \mathrm{mM}$ glucose or $10 \mu \mathrm{g}$ LPS treatment substantially significantly increased the level of cleaved caspase-3, decreased the level of KCNJ11 and the glucose or LPS effects were almost completely increased by the siRNA of KCNJ11. Data are presented as mean $\pm \mathrm{SD}(n=3) . * P<0.05, * * P<0.01$ in two-tailed Student's $t$-test. 

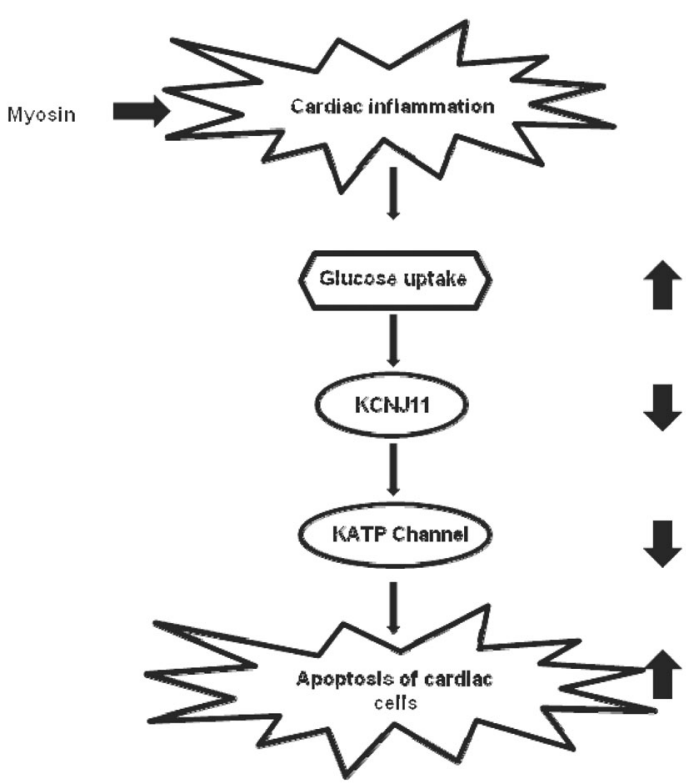

Figure 5. Estimated apoptosis pathway in the EAM model.

KATP channel is closed and $\mathrm{Ca}^{2+}$ enters into the cell, resulting in ER (endoplasmic reticulum) stress and membrane depolarization ${ }^{11,14-16,19,20}$. Studies also showed that ER stress occurs in EAM and KCNJ11 inactivation causes heart diseases ${ }^{22,34,35}$.

$\mathrm{Ca}^{2+}$-calmodulin-dependent proteolytic enzyme II (CaMKII) is a serine/threonine protein kinase that phosphorylates multiple excitation-contraction binding proteins $^{36-41}$. CaMKII is important in the pathogenesis of hypertrophy and heart dysfunction ${ }^{42}$. In the present study, we found that EAM tissues have elevated levels of glucose, reduced expression of $\mathrm{KCNJ} 11$ and increased expression of CaMKII. Using cultured cardiac cells, we also verified that LPS- or glucose-induced reduction of cardiac cell viability was attenuated when the cells were co-treated with KATP openers (DZ and Pin). Consistently, the $\mathrm{Ca}^{2+}$ chelate (BAPTA-AM) treatment suppressed cell death induced by LPS and high glucose, and this effect was further elevated by combined treatment with KATP openers. The immunoblot assay of cleaved caspase- 3 also revealed that the treatment using LPS or high glucose decreases KCNJ11 expression in cultured H9c2 cardiac cells. Furthermore, siRNA-mediated depletion of KCNJ11 was identified to promote apoptosis induced by LPS or glucose.

In a previous study, we have reported that apoptosis progresses in the heart tissue of the EAM model when Aldoart1 gene was expressed ${ }^{23}$. Aldoart1 is known as a protein of the fructose-1,6-bisphosphate aldolase family, and a gene related in glycolysis and gluconeogenesis ${ }^{23}$. When the H9c2 cardiac cells were treated with glucose, the Aldoart1 expression level was found to increase, including apoptosis in the $\mathrm{H} 9 \mathrm{c} 2$ cells $^{23}$. In addition, when the level of glucose increase in $\mathrm{H} 9 \mathrm{c} 2$ cardiac cells, it has an impact on KCNJ11 gene expression, which results in apoptosis. We assume that the two genes interact with each other by a mediator, directly or indirectly.

This study demonstrates that KCNJ11 expression is downregulated under inflammation and/or high glucose conditions, and its inactivation facilitates cardiac cell apoptosis and thereby contributes to myocarditis development. Figure 5 shows the model pathway.

\section{Conclusion}

We conclude that when KCNJ11 was inhibited, apoptosis occurs in heart cells, which affects the development of myocarditis.

Conflict of interest: The authors declare no competing financial interests.

1. Andreoletti, L., Leveque, N., Boulagnon, C., Brasselet, C. and Fornes, P., Viral causes of human myocarditis. Arch. Cardiovasc. Dis., 2009, 102, 559-568.

2. Pollack, A., Kontorovich, A. R., Fuster, V. and Dec, G. W., Viral myocarditis - diagnosis, treatment options, and current controversies. Nature Rev. Cardiol., 2015, 12, 670-680.

3. Smith, S. C., Autoimmune myocarditis. Current Protocols in Immunology, 2001, Chapter 15, Unit 15 14, 15.14.1-15.14.19.

4. Kodama, M. et al., Characteristics of giant cells and factors related to the formation of giant cells in myocarditis. Circ. Res., 1991, 69, 1042-1050.

5. Rose, N. R. and Hill, S. L., Autoimmune myocarditis. Int J. Cardiol., 1996, 54, 171-175.

6. Hu, F. et al., Effects of 1, 25-dihydroxyvitamin D3 on experimental autoimmune myocarditis in mice. Cell Physiol. Biochem., 2016, 38, 2219-2229.

7. Iurlaro, R. and Munoz-Pinedo, C., Cell death induced by endoplasmic reticulum stress. FEBS J., 2016, 283, 2640-2652.

8. Gogvadze, V., Orrenius, S. and Zhivotovsky, B., Multiple pathways of cytochrome $c$ release from mitochondria in apoptosis. Biochim. Biophys. Acta, 2006, 1757, 639-647.

9. Chen, Q., Gong, B. and Almasan, A., Distinct stages of cytochrome $c$ release from mitochondria: evidence for a feedback amplification loop linking caspase activation to mitochondrial dysfunction in genotoxic stress induced apoptosis. Cell Death Differ., 2000, 7, 227-233.

10. Rizzuto, R. et al., Calcium and apoptosis: facts and hypotheses. Oncogene, 2003, 22, 8619-8627.

11. Pinton, P., Giorgi, C., Siviero, R., Zecchini, E. and Rizzuto, R., Calcium and apoptosis: ER-mitochondria $\mathrm{Ca}^{2+}$ transfer in the control of apoptosis. Oncogene, 2008, 27, 6407-6418.

12. Yu, T., Sheu, S. S., Robotham, J. L. and Yoon, Y., Mitochondrial fission mediates high glucose-induced cell death through elevated production of reactive oxygen species. Cardiovasc. Res., 2008, 79, 341-351.

13. Chen, M., Wang, W., Ma, J., Ye, P. and Wang, K., High glucose induces mitochondrial dysfunction and apoptosis in human retinal pigment epithelium cells via promoting SOCS1 and Fas/FasL signaling. Cytokine, 2016, 78, 94-102.

14. La-Baj, B., Molecular biology of adenosine triphosphate-sensitive potassium channels. Endocr. Rev., 1999, 20, 101-135.

15. Joseph, C., Koster, M. A. P. and Nichols, C. G., Perspectives in diabetes diabetes and insulin secretion. The ATP-sensitive $\mathrm{K}^{+}$ channel (KATP) connection. Diabetes, 2005, 54, 3065-3072. 
16. Ashcroft, F. M., ATP-sensitive potassium channelopathies: focus on insulin secretion. J. Clin. Invest., 2005, 115, 2047-2058.

17. Liu, G. J. et al., ATP-sensitive potassium channels induced in liver cells after transfection with insulin cDNA and the GLUT2 transporter regulate glucose-stimulated insulin secretion. FASEB J., 2003, 17, 1682-1684.

18. Ashcroft, F. M., Puljung, M. C. and Vedovato, N., Neonatal diabetes and the KATP channel from mutation to therapy. Trends Endocrinol. Metab., 2017, 28, 377-387.

19. Rolland, J.-F., Henquin, J.-C. and Gilon, P., Feedback control of the ATP-sensitive $\mathrm{K}^{+}$current by cytosolic $\mathrm{Ca}^{2+}$ contributes to oscillations of the membrane potential in pancreatic cells. Diabetes, 2002, 51, 376-384.

20. Nakano, K., Suga, S., Takeo, T., Ogawa, Y., Suda, T., Kanno, T. and Wakui, M., Intracellular $\mathrm{Ca}^{2}$ modulation of ATP-sensitive $\mathrm{K}^{+}$ channel activity in acetylcholine-induced activation of rat pancreatic-beta cells. Endocrinology, 2002, 143, 569-576.

21. Kane, G. C. et al., KCNJ11 gene knockout of the Kir6.2 KATP channel causes maladaptive remodeling and heart failure in hypertension. Hum. Mol. Genet., 2006, 15, 2285-2297.

22. Liang, W. et al., The opening of ATP-sensitive $\mathrm{K}^{+}$channels protects $\mathrm{H} 9 \mathrm{c} 2$ cardiac cells against the high glucose-induced injury and inflammation by inhibiting the ROS-TLR4-necroptosis pathway. Cell Physiol. Biochem., 2017, 41, 1020-1034.

23. Choi, S. M. et al., Elevated aldolase 1A, retrogene 1 expression induces cardiac apoptosis in rat experimental autoimmune myocarditis model. Can. J. Physiol. Pharm., 2020, 98, 373-382.

24. Meiboom, S. and Gill, D., Modified spin-echo method for measuring nuclear relaxation times. Rev. Sci. Instrum., 1958, 29, 688691.

25. Jiang, Chun-Ying, Yang, Kang-Min, Yang, Liu, Miao, Zhao-Xia, Wang, Ying-Hong and Zhu, Hai-Bo, A ${ }^{1} \mathrm{H}$ NMR-based metabonomic investigation of time-related metabolic trajectories of the plasma, urine, and liver extracts of hyperlipidemic hamsters. PLoS ONE, 2013, 8, e66786.

26. Merrifield, C. A. et al., A metabolic system-wide characterization of the pig: a model for human physiology. Mol. BioSyst., 2011, 7, 2577-2588.

27. Chuichi Kawai, M., From myocarditis to cardiomyopathy: mechanisms of inflammation and cell death: learning from the past for the future. Circulation, 1999, 99, 1091-1100.

28. Wu, B. et al., The impact of circulating mitochondrial DNA on cardiomyocyte apoptosis and myocardial injury after TLR4 activation in experimental autoimmune myocarditis. Cell. Physiol. Biochem., 2017, 42, 713-728.

29. Fu, C., Dai, X., Yang, Y., Lin, M., Cai, Y. and Cai, S., Dexmedetomidine attenuates lipopolysaccharide-induced acute lung injury by inhibiting oxidative stress, mitochondrial dysfunction and apoptosis in rats. Mol. Med. Rep., 2017, 15, 131-138.

30. Yucel, G. et al., Lipopolysaccharides induced inflammatory responses and electrophysiological dysfunctions in human-induced pluripotent stem cell derived cardiomyocytes. Sci. Rep., 2017, 7, 2935.

31. Rogatzki, M. J., Ferguson, B. S., Goodwin, M. L. and Gladden, L. B., Lactate is always the end product of glycolysis. Front. Neurosci., 2015, 9, 22.

32. Kawase, T. et al., Validation of lactate level as a predictor of early mortality in acute decompensated heart failure patients who entered intensive care unit. J. Cardiol., 2015, 65, 164-170.

33. Mishra, R., Emancipator, S. N., Kern, T. and Simonson, M. S., High glucose evokes an intrinsic proapoptotic signaling pathway in mesangial cells. Kidney Int., 2005, 67, 82-93.

34. Tinker, A., Aziz, Q. and Thomas, A., The role of ATP-sensitive potassium channels in cellular function and protection in the cardiovascular system. Br. J. Pharmacol., 2014, 171, 12-23.

35. Richer, M. J. and Horwitz, M. S., The innate immune response: an important partner in shaping coxsackievirus-mediated autoimmunity. J. Innate. Immun., 2009, 1, 421-434.

36. Salas, M. A. et al., The signalling pathway of CaMKII-mediated apoptosis and necrosis in the ischemia/reperfusion injury. J. Mol. Cell Cardiol., 2010, 48, 1298-1306.

37. Anderson, M. E., Braun, A. P., Schulman, H. and Premack, B. A., Multifunctional $\mathrm{Ca}^{2+} /$ calmodulin-dependent protein kinase mediates $\mathrm{Ca}(2+)$-induced enhancement of the L-type $\mathrm{Ca}^{2+}$ current in rabbit ventricular myocytes. Circ. Res., 1994, 75, 854-861.

38. Mundiña-Weilenmann, C., Vittone, L., Ortale, M., de Cingolani, G. C. and Mattiazzi, A., Immunodetection of phosphorylation sites gives new insights into the mechanisms underlying phospholamban phosphorylation in the intact heart. J. Biol. Chem., 1996, 271, 33561-33567.

39. Maier, L. S. and Bers, D. M., Calcium, calmodulin, and calciumcalmodulin kinase II: Heartbeat to heartbeat and beyond. J. Mol. Cell Cardiol., 2002, 34, 919-939.

40. Ferrero, P. et al., $\mathrm{Ca}^{2+} /$ calmodulin kinase II increases ryanodine binding and $\mathrm{Ca}^{2+}$-induced sarcoplasmic reticulum $\mathrm{Ca}^{2+}$ release kinetics during beta-adrenergic stimulation. J. Mol. Cell Cardiol., 2007, 43, 281-291.

41. MacDonnell, S. M. et al., Adrenergic regulation of cardiac contractility does not involve phosphorylation of the cardiac ryanodine receptor at serine 2808. Circ. Res., 2008, 102, e65-e72.

42. Zhang, T. and Brown, J. H., Role of $\mathrm{Ca}^{2+} /$ calmodulin-dependent protein kinase II in cardiac hypertrophy and heart failure. Cardiovasc. Res., 2004, 63, 476-486.

ACKNOWLEDGEMENTS. We thank H. Moon (Korea Basic Science Institute) for developing the EAM rat model. This work was supported by the KBSI Grant (no. E38300).

Received 30 August 2019; revised accepted 12 June 2020

doi: $10.18520 / \mathrm{cs} / \mathrm{v} 119 / \mathrm{i} 7 / 1106-1112$ 